\title{
The hydrodynamical structure of circumstellar envelopes around low mass-loss rate, low outflow velocity AGB stars ${ }^{\star}$
}

\author{
J. M. Winters ${ }^{1}$, T. Le Bertre ${ }^{2}$, L.-Å. Nyman ${ }^{3}$, A. Omont ${ }^{4}$, and K. S. Jeong ${ }^{2}$ \\ 1 Max-Planck-Institut für Radioastronomie, Auf dem Hügel 69, 53121 Bonn, Germany \\ 2 DEMIRM, UMR 8540, Observatoire de Paris, 61 av. de l'Observatoire, 75014 Paris, France \\ 3 Swedish-ESO Submillimetre Telescope, European Southern Observatory, Casilla 19001, Santiago 19, Chile \\ 4 Institut d'Astrophysique de Paris, CNRS, 98bis Boulevard Arago, 75014 Paris, France
}

Received 23 November 2001 / Accepted 3 April 2002

\begin{abstract}
Recent hydrodynamical models (Winters et al. 2000b) allow the existence of pulsating, large velocity amplitude Asymptotic Giant Branch (AGB) stars with tenuous dusty circumstellar shells, which produce low mass-loss rates and simultaneously low wind velocities. The Semi-regular long-period variable $\mathrm{L}^{2} \mathrm{Pup}$ shows a broad $\mathrm{SiO}$ maser feature at $86 \mathrm{GHz}(v=1, J=2-1)$, indicating an outward velocity of the material close to the stellar photosphere of at least $10 \mathrm{~km} \mathrm{~s}^{-1}$, and narrow CO $(J=2-1)$ and $(1-0)$ line profiles indicating an outflow velocity of the material in the circumstellar shell of only about $3 \mathrm{~km} \mathrm{~s}^{-1}$. This can be explained in terms of our hydrodynamical models which provide large velocities in the shocked stellar atmosphere and low velocities of the circumstellar outflow.
\end{abstract}

Key words. hydrodynamics - stars: mass-loss - stars: AGB and post-AGB - stars: individual: $\mathrm{L}^{2}$ Pup stars: winds, outflows - radio lines: stars

\section{Introduction}

A time dependent hydrodynamic description of dustforming circumstellar shells around pulsating AGB stars has been developed by Fleischer et al. (1992) and subsequently has been applied to successfully model different observed data obtained from high mass-loss rate AGB stars (e.g., Winters et al. 1997; Winters et al. 2000a). In Winters et al. (2000b) we presented results for a large grid of models, covering the range of parameters relevant for AGB stars. We found two different classes of models, depending on the radiative acceleration produced in the dust formation region. For values of the radiative acceleration $\alpha=g^{\mathrm{rad}} / g^{\text {grav }}>1$, we find a layered structure of the circumstellar dust shell, which is expanding at typical velocities of $5 \mathrm{~km} \mathrm{~s}^{-1} \lesssim v_{\exp } \lesssim 40 \mathrm{~km} \mathrm{~s}^{-1}$ and carrying mass-loss rates in the range $3 \times 10^{-7} M_{\odot} \mathrm{yr}^{-1} \lesssim \dot{M} \lesssim$ $10^{-4} M_{\odot} \mathrm{yr}^{-1}$, typical of dusty, mass-losing AGB stars (class A). In the models of the second class B, i.e. for $\alpha<1$, the circumstellar shell displays a rather smooth hydrodynamic- and dust structure, closely resembling a

Send offprint requests to: J. M. Winters,

e-mail: jwinters@mpifr-bonn.mpg.de

* Based on observations obtained at the European Southern Observatory, La Silla, Chile. stationary outflow. These models are characterized by low expansion velocities, $v_{\exp } \lesssim 5 \mathrm{~km} \mathrm{~s}^{-1}$ and simultaneously low mass-loss rates, $\dot{M} \lesssim 3 \times 10^{-7} M_{\odot} \mathrm{yr}^{-1}$. In order to check, whether these theoretically predicted dust shells of the second class B are realized in nature, we started a CO $(J=2-1)$ survey of sources which show only a weak infrared excess.

For this survey, we selected a set of targets which, from their infrared photometric indices (Epchtein et al. 1987), appear to be late-type giants with weak circumstellar dust shells. Here, we present observations of one source of our sample, $\mathrm{L}^{2}$ Pup, which give support to the existence in nature of AGB objects which correspond to the B-type models.

The aim of the present paper is to relate $\mathrm{SiO}$ and CO data, which seem conflicting to each other and to give a physical interpretation of the velocity structure suggested by these observations in terms of independently developed hydrodynamic model calculations. Thereby, evidence is provided that a theoretically predicted physical mechanism is in fact realized in nature.

\section{Presentation of the source}

$\mathrm{L}^{2} \mathrm{Pup}(=\mathrm{IRAS07120}-4433=\mathrm{HD} 56096)$ is a Semi-regular variable of type $\mathrm{SRb}$ with a pulsation period of 141 days 
(Kholopov et al. 1985). Its spectral type is M5IIIe-M6IIIe and its amplitude in the $K$-band is $\Delta K \approx 0.29 \mathrm{mag}$ from which a bolometric amplitude $\Delta m_{\text {bol }}=0.38$ mag has been derived by Whitelock et al. (2000). The Hipparcos satellite measured a parallax of $16.46 \pm 1.27$ mas which translates to a distance of $\mathrm{L}^{2}$ Pup of $d=61 \pm 5 \mathrm{pc}$.

The near-infrared photometry by Fouqué et al. (1992) shows a weak infrared excess $\left(K-L^{\prime} \sim 0.6\right)$. At longer wavelengths, IRAS data shows also a weak excess; in a [1225, 25-60] color diagram $\mathrm{L}^{2}$ Pup is found close to the border between regions I and II in the scheme of van der Veen \& Habing (1988).

The bolometric magnitude of $\mathrm{L}^{2}$ Pup can be determined from the the near-infrared photometry $\left(K, L^{\prime}=\right.$ $-2.49,-3.06$; Fouqué et al. 1992) and the bolometric correction in the $K$-band as given in Le Bertre et al. (2001):

$m_{\text {bol }}=K+B C_{K}=-2.49+2.70=0.21$.

Using the distance derived from the Hipparcos parallax, we obtain for the luminosity:

$L_{\star}\left(d=61_{-5}^{+5} \mathrm{pc}\right)=2430_{-385}^{+410} L_{\odot}$.

Perrin et al. (1998) give an effective temperature $T_{\text {eff }}=$ $3420 \mathrm{~K}$ for a M5III spectral type and $T_{\text {eff }}=3243 \pm 79 \mathrm{~K}$ for M6III. Dumm \& Schild (1998) derive an effective temperature $T_{\text {eff }}=3380 \mathrm{~K}$, a radius $R_{\star}=126 R_{\odot}$ and a mass $M_{\star}=1.7 M_{\odot}$ for $\mathrm{L}^{2}$ Pup.

The estimated temperature and luminosity suggest, that $\mathrm{L}^{2}$ Pup is very likely an AGB star, a conclusion which is supported by the probable detection of Tc in its atmosphere by Little et al. (1987) and Lebzelter \& Hron (1999).

\section{Observations}

We observed $\mathrm{L}^{2}$ Pup in $\mathrm{CO}(2-1)$ and $\mathrm{CO}(1-0)$ and $\mathrm{SiO}$ $(v=1, J=2-1)$ with the SEST on September 28, 2001. The receivers were SIS receivers and typical system temperatures above the atmosphere were 300 and $500 \mathrm{~K}$, respectively. The beam width of the telescope is $50^{\prime \prime}$ at $3 \mathrm{~mm}$ and $23^{\prime \prime}$ at $1.3 \mathrm{~mm}$. The observations were done in a dual beam switch mode with a beam throw of $11.5^{\prime}$. The intensities reported here are given in main beam brightness temperatures, $T_{\mathrm{mb}}$, which is the chopper wheel corrected antenna temperature, $T_{\mathrm{A}}^{*}$, divided by the main beam efficiency, $\eta_{\mathrm{mb}}$. The main beam efficiency is 0.7 and 0.5 at $3 \mathrm{~mm}$ and $1.3 \mathrm{~mm}$, respectively. For the CO observations three acousto optical spectrometers were used simultaneously, one high resolution (HRS) with a bandwidth of $86 \mathrm{MHz}$, a channel separation of $43 \mathrm{kHz}$, and a resolution of $80 \mathrm{kHz}$, the other two are low resolution spectrometers (LRS) with bandwidths of about $1 \mathrm{GHz}$, channel separations of $0.7 \mathrm{MHz}$, and resolutions of $1.4 \mathrm{MHz}$. The $\mathrm{SiO}$ maser observations were done just before the CO observations and used to check the pointing of the telescope. These observations were done in a single beam switch mode and only the HRS was used. The profiles are shown in Figs. 1 and 2.
Table 1. Results for the CO line profiles.

\begin{tabular}{lccccc}
\hline \hline $\begin{array}{l}\text { Line } \\
\text { CO }\end{array}$ & $\begin{array}{c}v_{\text {lsr }} \\
\mathrm{km} \mathrm{s}^{-1}\end{array}$ & $\begin{array}{c}v_{\exp } \\
\mathrm{km} \mathrm{s}^{-1}\end{array}$ & $\begin{array}{c}T_{\mathrm{mb}} \\
\mathrm{K}\end{array}$ & $\begin{array}{c}\mathrm{rms} \\
\mathrm{K}\end{array}$ & $\begin{array}{c}\int T(v) \mathrm{d} v \\
\mathrm{~K}\end{array} \mathrm{~km} \mathrm{~s}^{-1}$ \\
\hline$(1-0)$ & 32 & 3.5 & 0.074 & 0.017 & 0.32 \\
$(2-1)_{\text {LRS }}$ & 33 & 3 & 0.72 & 0.020 & 2.96 \\
$(2-1)_{\text {HRS }}$ & 33 & 3 & 0.78 & 0.025 & 3.19 \\
\hline
\end{tabular}

In order to determine the stellar velocity, $v_{\star}\left(=v_{\text {lsr }}\right)$, the outflow velocity of the wind, $v_{\exp }$, and the main beam brightness temperature, $T_{\mathrm{mb}}$, we fit the CO line profiles (after subtraction of a linear baseline) by a parabola (Olofsson et al. 1993):

$T(v)=T_{\mathrm{mb}}\left[1-\left(\frac{v-v_{\star}}{v_{\exp }}\right)^{2}\right]$.

The integrated intensity is determined by integrating the observed intensity in the interval $\left[v_{\star}-v_{\exp }, v_{\star}+v_{\exp }\right]$. Our results are summarized in Table 1.

The SiO feature was particularly bright in our observation $\left(F_{\text {peak }}=76 \mathrm{Jy}\right)$ as compared to an earlier observation obtained on March 16, 2001, when the maser peak was at less than $25 \mathrm{Jy}$. The $\mathrm{SiO}$ line shows several bright spikes, notably two at $42 \mathrm{~km} \mathrm{~s}^{-1}$ and $26 \mathrm{~km} \mathrm{~s}^{-1}$, i.e. at $-9 \mathrm{~km} \mathrm{~s}^{-1}$ and $7 \mathrm{~km} \mathrm{~s}^{-1}$ with respect to $v_{\star}=33 \mathrm{~km} \mathrm{~s}^{-1}$ (see Fig. 1). The $\mathrm{SiO} 86 \mathrm{GHz}$ line in $\mathrm{L}^{2}$ Pup was first detected by Haikala (1990), and at this time the line showed 3 peaks at $32 \mathrm{~km} \mathrm{~s}^{-1}, 38 \mathrm{~km} \mathrm{~s}^{-1}$, and $42 \mathrm{~km} \mathrm{~s}^{-1}$, respectively, with peak flux densities of $65 \mathrm{Jy}, 60 \mathrm{Jy}$, and $35 \mathrm{Jy}$. It was re-observed by Haikala et al. (1994) and, at that time, showed only two peaks at $27 \mathrm{~km} \mathrm{~s}^{-1}$ and $32 \mathrm{~km} \mathrm{~s}^{-1}$ with peak intensities of $25 \mathrm{Jy}$ and $33 \mathrm{Jy}$, respectively.

\section{Interpretation}

The $\mathrm{SiO}$ masers are supposed to arise very close to the star in the pulsating atmosphere, probably between 1.3 and $2 R_{\star}$ (e.g., Greenhill et al. 1995; Boboltz et al. 1997), whereas the low excitation CO rotational lines arise from the expanding circumstellar shell.

From Fig. 1 it is obvious, that the $\mathrm{SiO}$ maser line from $\mathrm{L}^{2}$ Pup indicates much higher velocities than the CO rotational lines, i.e. the material close to the stellar photosphere is moving at higher velocities than the material in the circumstellar shell. This behavior was previously predicted by hydrodynamical wind models for dust forming circumstellar shells of pulsating AGB stars in the case of low mass-loss rates (Winters et al. 2000b).

These models comprise the simultaneous solution of the coupled equation system describing time-dependent hydrodynamics, (equilibrium) chemistry, dust formation, -growth and -evaporation, and the (continuum) radiative transfer problem. The solution of this equation system is completely determined by the prescription of the 4 stellar 


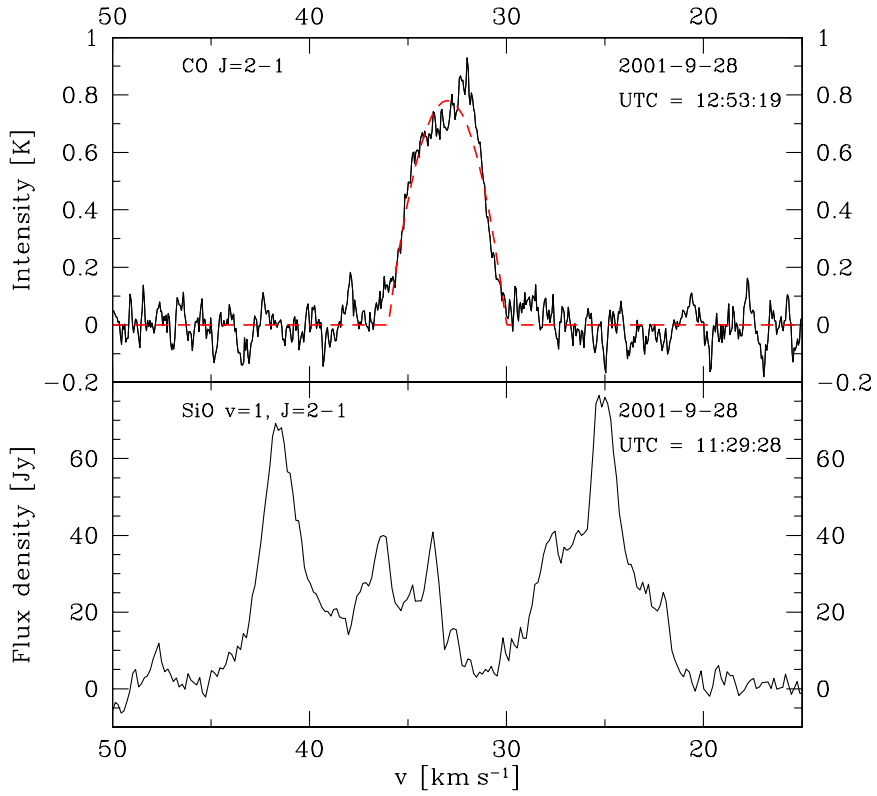

Fig. 1. High-resolution CO (2-1) line profile (upper panel, solid line; the dashed line represents the fit according to Eq. (1)) and $\mathrm{SiO}$ maser line at $86 \mathrm{GHz}$ (lower panel). Note, that the $\mathrm{SiO}$ line indicates much larger velocities than the $\mathrm{CO}$ line.

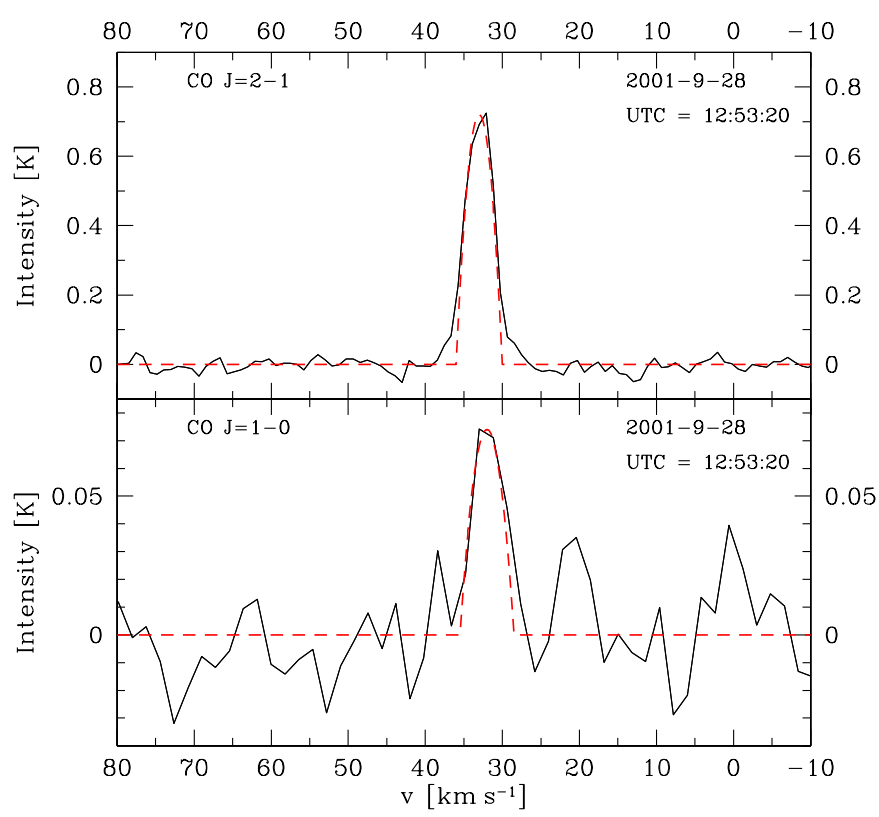

Fig. 2. Low-resolution CO (2-1) line profile (upper panel) and CO (1-0) line profile (lower panel). The dashed lines represent the fits according to Eq. (1)).

parameters stellar mass $M_{\star}$, stellar luminosity $L_{\star}$, stellar temperature $T_{\star}$, and photospheric element abundances $\epsilon_{\mathrm{i}}$, augmented by two additional quantities characterizing the period $P$ and velocity amplitude $\Delta v_{\mathrm{p}}$ of the stellar pulsation. Details of these model calculations can be found in Fleischer et al. (1992) and Winters et al. (1997, 2000b). By this approach, the spatial and temporal structure of the dust shell as well as average quantities like terminal wind velocity, mass-loss rate, gas-to-dust ratio, and optical depths are a result of the calculation. Our treatment represents a self-consistent solution to the problem, there is no freedom to prescribe any velocity-, temperature-, or density structure or the composition, radial distribution or size-distribution of the dust component. This is in contrast to other approaches, which aim at fitting, e.g., an observed spectral energy distribution or a spectral line by adjusting free model parameters like the density gradient, outflow velocity, dust particle size (distribution), optical depth, or the composition of the dust grains. Also, as dust nucleation and -growth is treated on a physical basis here, there is no freedom to choose a dust condensation temperature or a parameterization of the radiation pressure on dust as done, e.g., in the pioneering hydrodynamical models presented by Wood (1979) and Bowen (1988).

We like to stress, that these models have not been designed to fit $\mathrm{L}^{2}$ Pup or any other low mass-loss rate AGB star. In particular, the model discussed in the following has been calculated for a carbon-rich element mixture. However, the general mechanism leading to the typical wind structure of a low mass-loss rate, low velocity (B-type) model as displayed in Fig. 3 also applies to oxygen-rich situations (see Jeong 2000): the chemistry (O-rich or C-rich) does not change the topology of the solution space, i.e. the separation into A-type and B-type models. This topology is induced by the absolute value (larger or smaller than unity) of the radiative acceleration $\alpha$ in the sonic region, irrespective of the chemical composition of the dust grains responsible for $\alpha$. We therefore believe that the characteristics of the B-type model discussed here are general and are also applicable to the case of the oxygen-rich source $L^{2}$ Pup. Still, we expect that the chemistry has some influence on the exact structure of an individual model and (for the same set of parameters) on the exact values of resulting average quantities like the dust-to-gas ratio, mass-loss rate, or the outflow velocity.

In the B-type models, pulsation of the star dominates the hydrodynamical structure of the inner atmosphere. Only small amounts of dust form, which, together with the comparably low luminosity, leads to only a small radiative acceleration of the material in the circumstellar shell. This B-type solution is favored by either low luminosities, high stellar temperatures, high stellar masses, short pulsation periods, low pulsation amplitudes, a small abundance of condensible material, or a combination of those (for details see Winters et al. 2000b). In Table 2, a selection of B-type models is compiled which gives examples of appropriate parameter combinations and typical wind characteristics.

A snapshot of the hydrodynamic structure of model w127 is shown in Fig. 3. The stellar pulsation induces strong shocks propagating through the stellar atmosphere which are rapidly damped within $5 R_{\star}$ from the star (upper panel of Fig. 3, solid line). Dust forms in the extended atmosphere, but the radiative acceleration $\alpha$ acting on the grains is not sufficient to drive a massive outflow, $\alpha$ stays below 1 (upper panel of Fig. 3, dashed line). Due to the small radiation pressure, the 
Table 2. Sample of B-type models from Winters et al. (2000b)

\begin{tabular}{lccccccccc}
\hline \hline No. & $\begin{array}{c}M_{\star} \\
{\left[M_{\odot}\right]}\end{array}$ & $\begin{array}{c}L_{0} \\
{\left[L_{\odot}\right]}\end{array}$ & $\begin{array}{c}T_{0} \\
{[\mathrm{~K}]}\end{array}$ & $\mathrm{C} / \mathrm{O}$ & $\begin{array}{c}P \\
{[\mathrm{~d}]}\end{array}$ & $\begin{array}{c}\Delta v_{\mathrm{p}} \\
{\left[\mathrm{km} \mathrm{s}^{-1}\right]}\end{array}$ & $\begin{array}{c}\dot{M} \\
{\left[M_{\odot} \mathrm{yr}^{-1}\right]}\end{array}$ & $\begin{array}{c}v_{\infty} \\
{\left[\mathrm{km} \mathrm{s}^{-1}\right]}\end{array}$ & $\begin{array}{c}\rho^{\mathrm{d}} / \rho^{\mathrm{g}} \\
10^{-3}\end{array}$ \\
\hline w54 & 1.2 & 12000 & 3000 & 1.30 & 400 & 5.0 & $1.3 \mathrm{e}-08$ & 1.6 & 0.8 \\
w147 & 1.0 & 10000 & 2600 & 1.20 & 650 & 2.0 & $6.9 \mathrm{e}-08$ & 1.5 & 0.6 \\
w148 & 1.0 & 10000 & 3300 & 1.80 & 650 & 2.0 & $7.2 \mathrm{e}-09$ & 1.5 & 0.8 \\
w128 & 0.8 & 7500 & 2600 & 1.30 & 200 & 5.0 & $5.4 \mathrm{e}-08$ & 2.4 & 0.6 \\
w127 & 0.8 & 5000 & 2600 & 1.30 & 250 & 5.0 & $1.2 \mathrm{e}-07$ & 2.4 & 0.8 \\
w118 & 0.8 & 7500 & 3000 & 1.50 & 104 & 5.0 & $3.2 \mathrm{e}-10$ & 4.2 & 0.0 \\
w107 & 0.8 & 7500 & 3000 & 1.50 & 104 & 2.0 & $3.2 \mathrm{e}-10$ & 4.2 & 0.0 \\
w105 & 1.0 & 9000 & 3000 & 1.30 & 400 & 5.0 & $8.5 \mathrm{e}-08$ & 1.7 & 0.6 \\
\hline
\end{tabular}

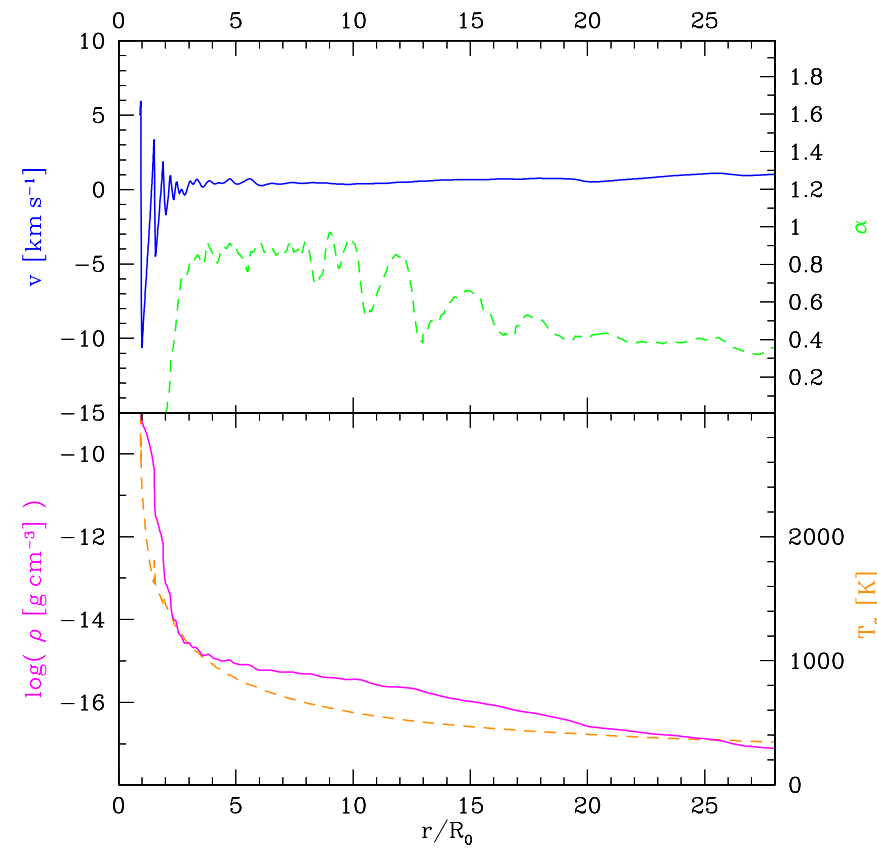

Fig. 3. Hydrodynamic structure of a B-type model (w127 from Winters et al. 2000b). Upper panel: hydrodynamic velocity $v$ (solid line, l.h.s. ordinate) and radiative acceleration $\alpha$ (dashed line, r.h.s. ordinate). Lower panel: mass density $\rho$ (solid line, l.h.s. ordinate) and gas temperature $T_{\mathrm{g}}$ (dashed line, r.h.s. ordinate)

gas is only weakly accelerated, and the expansion velocity reached in this type of models is less than $5 \mathrm{~km} \mathrm{~s}^{-1}$. In the inner part of the atmosphere, the shocks show maximum infall velocities of the material during the cycle of about $-11 \mathrm{~km} \mathrm{~s}^{-1}$ and maximum outward velocities during the cycle of about $7 \mathrm{~km} \mathrm{~s}^{-1}$. These model results are very much in agreement with the velocities observed in $\mathrm{L}^{2}$ Pup. The model produces a mass-loss rate $\dot{M}=1.2 \times 10^{-7} M_{\odot} \mathrm{yr}^{-1}$, a terminal expansion velocity $v_{\text {exp }}=2.4 \mathrm{~km} \mathrm{~s}^{-1}$, a dust-to-gas ratio $\rho^{\mathrm{d}} / \rho^{\mathrm{g}}=0.8 \times 10^{-3}$, and a luminosity amplitude of $0.34 \mathrm{mag}$.
The luminosity of $\mathrm{L}^{2} \mathrm{Pup}$ is lower and the temperature is higher than in any of the hydro-models in Winters et al. (2000b). Since the mass-loss rate as well as the outflow velocity of our models decrease with increasing temperature and decreasing luminosity (Arndt et al. 1997; Wachter et al. 2002) we conclude that an object like $\mathrm{L}^{2}$ Pup must surely have extremely low $v_{\exp }$ and $\dot{M}$. Combined with the fact that $\mathrm{L}^{2}$ Pup is variable this implies that $\mathrm{L}^{2}$ Pup should in fact be an object corresponding to the B-type models.

\section{Discussion}

$\mathrm{L}^{2}$ Pup has been detected previously in CO (1-0) by Kerschbaum et al. (1996), and in CO (2-1) and (3-2) by Kerschbaum \& Olofsson (1999). Their observation gives $v_{\mathrm{lsr}}=33.1 \mathrm{~km} \mathrm{~s}^{-1}, v_{\exp }=1.9 \mathrm{~km} \mathrm{~s}^{-1}$, a peak main beam brightness temperature of $T_{\mathrm{mb}}=0.088 \mathrm{~K}$, and an integrated line intensity of $0.27 \mathrm{~K} \mathrm{~km} \mathrm{~s}^{-1}$ for the $\mathrm{CO}(1-0)$ line. Kerschbaum et al. (1996) estimate the mass-loss rate from $\mathrm{CO}(1-0)$ to $6.7 \times 10^{-10} M_{\odot} \mathrm{yr}^{-1}$ for a distance of $100 \mathrm{pc}$ (which translates to $2.5 \times 10^{-10} M_{\odot} \mathrm{yr}^{-1}$ at $61 \mathrm{pc}$ ).

To estimate the mass-loss rate from the CO (2-1) line, we use the expression given by Knapp \& Morris (1985) in a slightly modified version (Olofsson et al. 1993):

$$
\dot{M}=5.7 \times 10^{-20} \frac{T_{\mathrm{mb}} v_{\mathrm{exp}}^{2} d^{2} \Theta_{\mathrm{mb}}^{2}}{s(J) f_{0}^{0.85}} M_{\odot} \mathrm{yr}^{-1}
$$

where $\Theta_{\mathrm{mb}}$ is the full half power main beam width of the telescope $\left(23^{\prime \prime}\right.$ for the SEST at $\left.230 \mathrm{GHz}\right), s(J)=0.5$ for the $\mathrm{CO}(2-1)$ line, and $f_{0}$ is the $\mathrm{CO} / \mathrm{H}_{2}$ ratio (assumed to be $\left.2 \times 10^{-4}\right)$. From Eq. (2) we obtain a massloss rate for $\mathrm{L}^{2}$ Pup of $\dot{M}=2.2 \times 10^{-9} M_{\odot} \mathrm{yr}^{-1}$. Using the expressions given by Loup et al. (1993) (corrected for the $15 \mathrm{~m}$ diameter of the SEST) to determine consistently both, the mass-loss rate and the CO dissociation radius $R_{\mathrm{CO}}$ from the $\mathrm{CO}(1-0)$ line results in a mass-loss rate of $\dot{M}=4.5 \times 10^{-8} M_{\odot} \mathrm{yr}^{-1}$ and a $\mathrm{CO}$ radius of $R_{\mathrm{CO}}=1.3 \times 10^{16} \mathrm{~cm}$. Since the Loup et al. (1993) expressions do not take into account the infrared pumping of the 
Table 3. Maser and CO velocities observed in $\mathrm{L}^{2}$ Pup.

\begin{tabular}{llcl}
\hline \hline Molecule & frequency & $\begin{array}{c}\text { velocity spread } \\
\text { km s}^{-1}\end{array}$ & ref.* \\
\hline $\mathrm{SiO}$ & $86 \mathrm{GHz}$ & $21-43$ & $\mathrm{a}, \mathrm{b}, \mathrm{f}$ \\
$\mathrm{SiO}$ & $43 \mathrm{GHz}$ & $25-42$ & $\mathrm{c}$ \\
$\mathrm{H}_{2} \mathrm{O}$ & $22 \mathrm{GHz}$ & $28-37$ & $\mathrm{c}$ \\
$\mathrm{OH}$ & $1665 \mathrm{MHz}$ & $31-36$ & $\mathrm{~d}$ \\
$\mathrm{OH}$ & $1667 \mathrm{MHz}$ & $32-35$ & $\mathrm{~d}$ \\
$\mathrm{CO}$ & $115 / 230 \mathrm{GHz}$ & $30-36$ & e, f \\
\hline
\end{tabular}

* a: Haikala (1990), b: Haikala et al. (1994), c: Lépine et al. (1978), d: Dickinson et al. (1986), e: Kerschbaum et al. (1996), $\mathrm{f}$ : this work.

CO molecule which might be important in optically thin situations, this mass-loss rate has to be regarded as an upper limit. Therefore, the mass-loss rate and outflow velocity of $\mathrm{L}^{2} \mathrm{Pup}$ correspond to the typical values of B-type models, i.e. $\dot{M}<3 \times 10^{-7} M_{\odot} \mathrm{yr}^{-1}$ and $v_{\exp }<5 \mathrm{~km} \mathrm{~s}^{-1}$.

Estimates of the mass-loss rates derived from IR color indices should be handled with care for low mass-loss rate giants, since they rely on a "standard" expansion velocity of $15 \mathrm{kms}^{-1}$. In objects like $\mathrm{L}^{2}$ Pup these rates could therefore be overestimated by at least a factor $\sim 5$.

The velocity structure of $\mathrm{L}^{2}$ Pup can be further probed by observations of different masers, which are excited in different regions of the circumstellar shell. Lépine et al. (1978) present observations of the $\mathrm{H}_{2} \mathrm{O}$ maser at $22 \mathrm{GHz}$, showing two peaks separated by $8 \mathrm{~km} \mathrm{~s}^{-1}$ and centered on $33 \mathrm{~km} \mathrm{~s}^{-1}$, the stellar velocity (Table 3 ). This maser is supposed to form outside of the $\mathrm{SiO}$ masing region (e.g., Chapman \& Cohen 1986), in good agreement with its velocity being intermediate between the $\mathrm{SiO}$ and the $\mathrm{CO}$ velocity. $\mathrm{OH}$ main line masers at $1665 \mathrm{MHz}$ and $1667 \mathrm{MHz}$ have been detected in $\mathrm{L}^{2}$ Pup by Dickinson et al. (1986). The lines are centered at $33 \mathrm{~km} \mathrm{~s}^{-1}$ and $34 \mathrm{kms}^{-1}$, respectively, and extend from $31 \mathrm{~km} \mathrm{~s}^{-1}$ to $36 \mathrm{~km} \mathrm{~s}^{-1}$, and from $32 \mathrm{~km} \mathrm{~s}^{-1}$ to $35 \mathrm{~km} \mathrm{~s}^{-1}$, respectively. From these velocities, in the context of our models, it seems like the $\mathrm{OH}$ main line maser would arise outside of the $\mathrm{H}_{2} \mathrm{O}$ masing region. This behavior is different from the one reported by Chapman \& Cohen (1986) who find that for VX Sgr the $\mathrm{OH}$ main line masers are coextensive with the $\mathrm{H}_{2} \mathrm{O}$ masers. Unfortunately, $\mathrm{L}^{2}$ Pup was not detected in the $\mathrm{OH}$ satellite lines at a flux level of $0.15 \mathrm{Jy}$ (Dickinson et al. 1986), a result which was confirmed by Te Lintel Hekkert et al. (1991).

Very recently Jura et al. (2002) discussed the wind of $\mathrm{L}^{2}$ Pup on the basis of 11.7 and $17.9 \mu \mathrm{m}$ images obtained with the Keck I telescope, and on the basis of infrared light curves obtained by DIRBE. They find that the properties of the $\mathrm{L}^{2}$ Pup wind are consistent with those of our B-type models. They also argue that the star should undergo non-radial pulsations, a phenomenon that we cannot account for in our models which assume spherical symmetry. On the other hand the physical mechanisms operating in the wind regime should stay the same because matter is moving radially.

\section{Conclusions}

The radio observations obtained for $\mathrm{L}^{2}$ Pup support the appropriateness of our recent hydrodynamical calculations (Winters et al. 2000b) where the main driving mechanism of the circumstellar outflow is the stellar pulsation and where the dust plays only a secondary rôle. As suggested by Jura et al. (2002), and considering its close distance $(d \sim 61 \mathrm{pc})$ and its apparent brightness, $\mathrm{L}^{2}$ Pup could be taken as a prototype of red giants undergoing low massloss rates with low expansion velocities.

One of the difficulties encountered in the study of maser features in late-type giants arises from the lack of appropriate hydrodynamical models of their atmospheres. The diversity of the behaviors has long been a puzzle. The fact that we found 2 very different kinds of hydrostructures in our models may bring new light on the interpretation of stellar masers.

Our results further show that for objects with characteristics similar to the B-type models the mass-loss rate might be largely overestimated.

Acknowledgements. We thank Nicolas Epchtein for his continuous support of this project. We are grateful to the second referee, Dr. Yvonne Simis, for her insightful and considerate comments which helped to improve the presentation of the material. The calculations were performed on the Cray computers of the Konrad-Zuse-Zentrum für Informationstechnik Berlin. This work has been supported by the PROCOPE program under grants D/9822849 (DAAD) and F/01303ZA (MAE). This research has made use of the SIMBAD database, operated at CDS, Strasbourg, France and of NASA's Astrophysics Data System.

\section{References}

Arndt, T. U., Fleischer, A. J., \& Sedlmayr, E. 1997, A\&A, 327, 614

Boboltz, D. A., Diamond, P. J., \& Kemball, A. J. 1997, ApJ, 487, L47

Bowen, G. H. 1988, ApJ, 329, 299

Chapman, J. M. \& Cohen, R. J. 1986, MNRAS, 220, 513

Dickinson, D. F., Turner, B. E., Jewell, P. R., \& Benson, P. J. 1986, AJ, 92, 627

Dumm, T., \& Schild, H. 1998, New Astron., 3, 137

Epchtein, N., Le Bertre, T., Lépine, J. R. D., et al. 1987, A\&AS, 71, 39

Fleischer, A. J., Gauger, A., \& Sedlmayr, E. 1992, A\&A, 266, 321

Fouqué, P., Le Bertre, T., Epchtein, N., Guglielmo, F., \& Kerschbaum, F. 1992, A\&AS, 93, 151

Greenhill, L. J., Colomer, F., Moran, J. M., et al. 1995, ApJ, 449,365

Haikala, L. K. 1990, A\&AS, 85, 875 
Haikala, L. K., Nyman, L.-Å., \& Forsström, V. 1994, A\&AS, 103, 107

Jeong, K. S. 2000, Ph.D. Thesis, TU-Berlin, Germany

Jura, M., Chen, C., \& Plavchan, P. 2002, ApJ, 569, 964

Kerschbaum, F., \& Olofsson, H. 1999, A\&AS, 138, 299

Kerschbaum, F., Olofsson, H., \& Hron, J. 1996, A\&A, 311, 273

Kholopov, P. N., Samus', N. N., Frolov, M. S., et al. 1985, General Catalogue of Variable Stars, 4th ed. (Nauka, Moscow)

Knapp, G. R., \& Morris, M. 1985, ApJ, 292, 640

Le Bertre, T., Matsuura, M., Winters, J. M., et al. 2001, A\&A, 376, 997

Lebzelter, T., \& Hron, J. 1999, A\&A, 351, 533

Lépine, J. R. D., Le Squeren, A. M., \& Scalise, Jr. E. 1978, ApJ, 225, 869

Little, S. J., Little-Marenin, I. R., \& Bauer, W. H. 1987, AJ, 94, 981

Loup, C., Forveille, T., Omont, A., \& Paul, J. F. 1993, A\&AS, 99, 291
Olofsson, H., Eriksson, K., Gustafsson, B., \& Carlström, U. 1993, ApJS, 87, 267

Perrin, G., Coudé du Foresto, V., Ridgway, S. T., et al. 1998, A\&A, 331, 619

Te Lintel Hekkert, P., Caswell, J. L., Habing, H. J., et al. 1991, A\&AS, 90, 327

van der Veen, W. E. C. J., \& Habing, H. J. 1988, A\&A, 194, 125

Wachter, A. C., Schröder, K.-P., Winters, J. M., Arndt, T. U., \& Sedlmayr, E. 2002, A\&A, 384, 452

Whitelock, P., Marang, F., \& Feast, M. 2000, MNRAS, 319, 728

Winters, J. M., Fleischer, A. J., Le Bertre, T., \& Sedlmayr, E. 1997, A\&A, 326, 305

Winters, J. M., Keady, J. J., Gauger, A., \& Sada, P. V. 2000a, A\&A, 359, 651

Winters, J. M., Le Bertre, T., Jeong, K. S., Helling, C., \& Sedlmayr, E. 2000b, A\&A, 361, 641

Wood, P. R. 1979, ApJ, 227, 220 